Communications in Physics, Vol. 30, No. 3 (2020), pp. 301-310

DOI:10.15625/0868-3166/30/3/15080

\title{
EXACT MODE SHAPES OF T-SHAPED AND OVERHANG-SHAPED MICROCANTILEVERS
}

\author{
LE TRI DAT ${ }^{a, b, \dagger}$, VU LAN ${ }^{c}$ AND NGUYEN DUY VY ${ }^{a, b}$ \\ ${ }^{a}$ Laboratory of Applied Physics, Advanced Institute of Materials Science, \\ Ton Duc Thang University, Ho Chi Minh City 756636, Vietnam \\ ${ }^{b}$ Faculty of Applied Sciences, Ton Duc Thang University, Ho Chi Minh City 756636, Vietnam \\ ${ }^{c}$ Quang Trung High School, Dong Xoai, Binh Phuoc 831304, Vietnam \\ ${ }^{\dagger}$ E-mail: letridat@tdtu.edu.vn
}

Received 19 May 2020

Accepted for publication 6 July 2020

Published 15 August 2020

\begin{abstract}
Resonance frequencies and mode shapes of microcantilevers are of important interest in micro-mechanical systems for enhancing the functionality and applicable range of the cantilevers in vibration transducing, energy harvesting, and highly sensitive measurement. In this study, using the Euler-Bernoulli theory for beam, we figured out the exact mode shapes of cantilevers of varying widths such as the overhang-or T-shaped cantilevers. The obtained mode shapes have been shown to significantly deviate from the approximate forms of a rectangular cantilever that are commonly used in mechanics and physics. They were then used to figure out the resonance frequencies of the cantilever. The analytical solutions have been confirmed by using the finite element method simulations with very low deviation. This study suggested a method for correctly obtaining the resonance frequency of microcantilevers with complicated dimensions, such as the doubly clamped cantilever with the undercut, with the overhangs at the clamped positions, or with an attached mass in the middle.
\end{abstract}

Keywords: microcantilever, mode shape, analytical method, overhang-shaped, T-shaped.

Classification numbers: 07.79.Lh, 78.20.N-, 65.40.De. 


\section{INTRODUCTION}

Micromechanical cantilever is a branch of sensor classes which is based on the response of the cantilever via changing its frequency and vibration amplitude with external effects. The commonly used structure of these sensors is a microbeam (or microcantilever) which can partly move or deflect when the surface stress is changed. Among them, the singly and doubly clamped cantilevers are widely adopted in experiments and also in industry due to the simplicity in fabrication and high adaptability in using. The cantilever-based sensors were in the heart of experiments in fundamental physics [1-4] and in detecting of bio-objects [5-8], chemical substances $[9,10]$, and physical properties [11,12]. In mass spectroscopy, to detect a mass $\Delta m$ that is adsorbed on the microcantilever surface, one usually examines the resonance frequency shift, which is related to $\Delta m$ via the formula [13],

$$
\Delta m=\frac{K}{4 \pi^{2}}\left(\frac{1}{f^{2}}-\frac{1}{f_{0}^{2}}\right),
$$

where $K$ is the mechanical rigidity (stiffness) of the cantilever and $f_{0}$ and $f$ are the frequencies before and after the adsorption occurs, respectively. In case without adsorption, the frequency $f_{0}$ is,

$$
f_{0}=\frac{1}{2 \pi}\left(\frac{\alpha_{n}}{L}\right)^{2} \sqrt{\frac{E I}{\rho A}},
$$

where $\alpha_{n}=1.875,4.694,7.854$, etc $\ldots$ are the roots of the characteristic equation $1+\cos \alpha \cosh \alpha=$ $0 . E$ and $I$ are the Young's modulus and the second moment of inertia, respectively. $A=w_{c} t_{c}$ is the cross-section, where $w_{c}$ is the width and $t_{c}$ is the thickness of the cantilever. $\rho$ is the mass density and $L$ is the cantilever length. Therefore, correctly determining the frequency before and after an adsorption is crucial to analyze the quantity and distribution of adsorbed mass. Theoretically, the frequencies of a cantilever can be determined from the Euler-Bernoulli theory of beam and the formula (2) is used. Nevertheless, in the fabrication, the cantilevers are usually of varying width or thickness with undercut effects or with an overhanging part at the clamped position. These parts could strongly alter the frequency from the frequency of an ideal rectangular-shaped beam [14]. The dynamics of these cantilevers are usually examined assuming the ideal mode shapes obtained from a rectangular cantilever. However, the results are correct if only the mode shapes are correct. Therefore, correctly figuring out the mode shapes is of important interest for the cantilever from both the theoretical and experimental viewpoint.

Furthermore, in recent studies the beams with various shapes such as T- $[15,16]$ or V-shaped cantilevers $[17,18]$ have attracted many researchers due to their wide application. However, it is challenging to theoretically obtain the frequency and deflection. Zhang et al. [19] explored the deflection and resonance frequency when the microcantilever dimensions were modified. In that work, the width of the microbeam was not constant and was dependent on the variable $x$ along the length; hence, finding the exact solution of the Euler-Bernoulli equation was a challenge. To overcome this difficulty, the solution was assumed as a function of polynomials. The calculated frequency revealed an involved dependence on the geometrical parameters. Furthermore, the solutions were lengthy in analytical form and strongly dependent on the polynomial approximation. In another study, Plaza et al. [15] considered the microcantilevers in an array to reduce the initial deflection; however, the frequency was known by only the experimental method. Therefore, the analytical formula for frequency of cantilevers with varying width is still of important interest. 

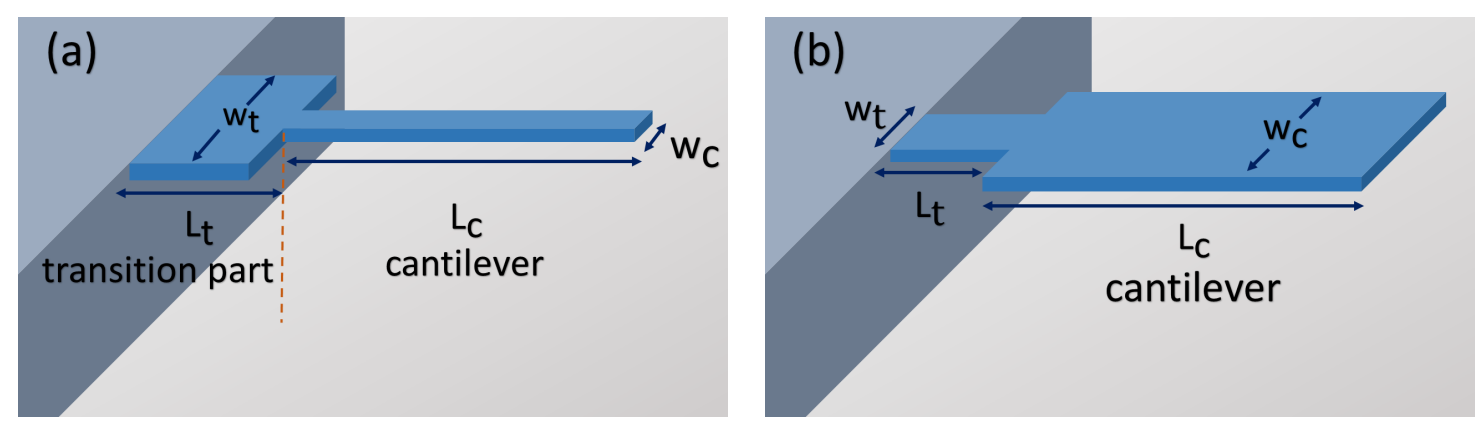

Fig. 1. Model of cantilevers with varying widths used in the calculation, (a) overhangand (b) T-shaped cantilever. The thickness $\left(t_{c}\right)$ is assumed to be uniform.

In this work, we figured out the exact mode shapes for cantilever structures such as T-shaped and overhang-shaped microcantilevers which are usually used in atomic force microscopes. The role of the length and width of the transition part, which is located between the anchor (the clamped position) and the cantilever part, was examined. Based on the exact mode shapes, the frequency can be obtained and corrected in comparison to the approximate values which were empirically used in literature. The analytical formula obtained from this work helped to correctly figure out the frequency of cantilever of similar structure, such as the douply clamped cantilever with overhangs of with an attached mass in the middle [20]. The obtained results can contribute to the exact measurement for these structures with many advantages such as determining the frequency of a cantilever in a array, a cantilever with undercut, or the T-shaped cantilevers that use the in-plan vibrational modes.

\section{CALCULATION MODEL AND METHOD}

We consider a cantilever with a transition part of width $w_{t}$ which connects the clamped position to the cantilever of width $w_{c}$, as shown in Fig. 1. The lengths of the transition and the cantilever are $L_{t}$ and $L_{c}$, respectively. For convenience, the reduced dimensions are used, $\kappa=w_{t} / w_{c}$ and $\eta=L_{t} / L$ with $L=L_{t}+L_{c}$ is the total length. To figure out the frequency and mode shape, we use the Euler-Bernoulli theory of beam

$$
\rho A(x) \frac{\partial^{2} u(x, t)}{\partial t^{2}}+\frac{\partial^{2}}{\partial x^{2}}\left[E I(x) \frac{\partial^{2} u(x, t)}{\partial x^{2}}\right]=0
$$

where $u(x, t)$ is the deflection at a position $x$ along the cantilever length at time $t$. The solution of Eq. (3) could be presented in the form $u(x, t)=u(x) e^{i \omega t}$. Inserting this form into the above equation, one obtains,

$$
\frac{d^{2}}{d x^{2}}\left[E I(x) \frac{d^{2} u(x)}{d x^{2}}\right]-\omega^{2} \rho A(x) u(x)=0,
$$

where $A(x)=w(x) t_{c}$. The varying width of beam is,

$$
w(x)= \begin{cases}w_{t} & \left(0<x \leq L_{t}\right) \\ w_{c} & \left(L_{t}<x \leq L\right)\end{cases}
$$


From Eq. (4) and using relation in Eqs. (5a) and (5b), the frequency has been rewritten as

$$
\begin{aligned}
\omega^{2} & =\frac{E t_{c}^{2}}{12 \rho} \frac{\int_{0}^{L} u(x) \frac{d^{2}}{d x^{2}}\left[w(x) \frac{d^{2} u(x)}{d x^{2}}\right] d x}{\int_{0}^{L} w(x) u^{2}(x) d x}=\frac{E t_{c}^{2}}{12 \rho} \frac{\int_{0}^{L_{t}} w_{t} u_{t}(x) \frac{d^{4} u_{t}(x)}{d x^{4}} d x+\int_{L_{t}}^{L} w_{c} u_{c}(x) \frac{d^{4} u_{c}(x)}{d x^{4}} d x}{\int_{0}^{L} w_{t} u_{t}^{2}(x) d x+\int_{L_{t}}^{L} w_{c} u_{c}^{2}(x) d x} \\
& =\frac{E t_{c}^{2}}{12 \rho} \frac{\psi_{t}+\frac{1}{\kappa} \psi_{c}}{\varphi_{t}+\frac{1}{\kappa} \varphi_{c}} .
\end{aligned}
$$

So we obtain

$$
\omega=\sqrt{\frac{E t_{c}^{2}}{12 \rho} \frac{\psi_{t}+\frac{1}{\kappa} \psi_{c}}{\varphi_{t}+\frac{1}{\kappa} \varphi_{c}}},
$$

where $\psi_{[t, c]}=\int u_{[t, c]}(x) u_{[t, c]}^{(4)}(x) d x$ and $\varphi_{[t, c]}=\int u_{[t, c]}^{2}(x) d x$. Here, $I(x)=\frac{1}{12} w(x) t_{c}^{3}$. The density of the beam is $\rho$ and the cross-section is $A(x)=w(x) t_{c}$. To figure out the frequency in Eq. (6), the explicit form of the mode shapes is required. The mode shapes of every part in the entire cantilever are derived by,

$$
\begin{aligned}
& u_{t}^{(4)}(x)-\beta_{t}^{4} u_{t}(x)=0, \\
& u_{c}^{(4)}(x)-\beta_{c}^{4} u_{c}(x)=0,
\end{aligned}
$$

where, $\beta_{t}^{4}=\frac{\omega^{2} \rho A_{t}}{E I_{t}}$ and $\beta_{c}^{4}=\frac{\omega^{2} \rho A_{c}}{E I_{c}}$. Noting that $\beta_{t}=\beta_{c}=\beta$, the general solutions are

\begin{tabular}{|c|c|c|}
\hline \multicolumn{2}{|c|}{ Boundary } & Continuous \\
\hline At $x=0$ & At $x=L$ & At $x=L_{t}$ \\
\hline$u_{t}(0)=0$ & & $u_{t}\left(L_{0}\right)=u_{c}\left(L_{0}\right)$ \\
\hline$u_{t}(0)=0$ & & $u_{t}^{\prime}\left(L_{0}\right)=u_{c}^{\prime}\left(L_{0}\right)$ \\
\hline & $u_{c}^{(2)}(L)=0$ & $\kappa u_{t}^{(2)}\left(L_{0}\right)=u_{c}^{(2)}\left(L_{0}\right)$ \\
\hline & $u_{c}^{(3)}(L)=0$ & $\kappa u_{t}^{(3)}\left(L_{t}\right)=u_{c}^{(3)}\left(L_{t}\right)$ \\
\hline
\end{tabular}

$$
\begin{aligned}
& u_{t}(x)=A_{1} \sin \beta x+B_{1} \cos \beta x+C_{1} \sinh \beta x+D_{1} \cosh \beta x \\
& u_{c}(x)=A_{2} \sin \beta x+B_{2} \cos \beta x+C_{2} \sinh \beta x+D_{2} \cosh \beta x .
\end{aligned}
$$

Table 1. Boundary and continuous conditions

To find out the eight coefficients of Eqs. (9) and (10), the boundary and continuity conditions are used (see more in Table 1). As a result, we obtained a matrix equation,

$$
K \cdot X=0,
$$


where $K$ is written as

$$
K=\left[\begin{array}{cccccccc}
0 & 1 & 0 & 1 & 0 & 0 & 0 & 0 \\
1 & 0 & 1 & 0 & 0 & 0 & 0 & 0 \\
0 & 0 & 0 & 0 & -\sin \alpha & -\cos \alpha & \sinh \alpha & \cosh \alpha \\
0 & 0 & 0 & 0 & -\cos \alpha & \sin \alpha & \cosh \alpha & \sinh \alpha \\
\sin \eta \alpha & \cos \eta \alpha & \sinh \eta \alpha & \cosh \eta \alpha & -\sin \eta \alpha & -\cos \eta \alpha & -\sinh \eta \alpha & -\cosh \eta \alpha \\
\cos \eta \alpha & -\sin \eta \alpha & \cosh \eta \alpha & \sinh \eta \alpha & -\cos \eta \alpha & \sin \eta \alpha & -\cosh \eta \alpha & -\sinh \eta \alpha \\
-\kappa \sin \eta \alpha & -\kappa \cos \eta \alpha & \kappa \sinh \eta \alpha & \kappa \cosh \eta \alpha & \sin \eta \alpha & \cos \eta \alpha & -\sinh \eta \alpha & -\cosh \eta \alpha \\
-\kappa \cos \eta \alpha & \kappa \sin \eta \alpha & \kappa \cosh \eta \alpha & \kappa \sinh \eta \alpha & \cos \eta \alpha & -\sin \eta \alpha & -\cosh \eta \alpha & -\sinh \eta \alpha
\end{array}\right]
$$

where, $\alpha=\beta L$ and $X=\left[\begin{array}{llllllll}A_{1} & B_{1} & C_{1} & D_{1} & A_{2} & B_{2} & C_{2} & D_{2}\end{array}\right]^{T} . K$ is a singular matrix; therefore, by solving $\operatorname{det} K=0$, one can obtain $\alpha$ (see, for example, Table 2),

$$
\begin{aligned}
\operatorname{det}[K]= & \left(\kappa^{2}-1\right)\{\cos [(1-\eta) \alpha] \cosh [(1-\eta) \alpha]+\cos \eta \alpha \cosh \eta \alpha\}+ \\
& +(1-\kappa)^{2}\{\cos [(1-\eta) \alpha] \cos \eta \alpha \cosh [(1-\eta) \alpha] \cosh \eta \alpha+1\} \\
& +2 \kappa(\cos \alpha \cosh \alpha+1) .
\end{aligned}
$$

It is worth to noting that, in a rectangular cantilever, we have $\kappa=1$ and the above equation returns to the characteristic equation $\cos \alpha \cosh \alpha+1=0$, which is well known in mechanics [21]. Now, solving the above equation with respect to $\alpha$, the following coefficients are obtained,

$$
\begin{aligned}
B_{1} & =\Gamma(\kappa, \eta, \alpha) A_{1}, \\
C_{1} & =-A_{1}, \\
D_{1} & =-\Gamma(\kappa, \eta, \alpha) A_{1}, \\
A_{2} & =H(\kappa, \eta, \alpha) A_{1}, \\
B_{2} & =E(\kappa, \eta, \alpha) A_{1}, \\
C_{2} & =\Omega(\kappa, \eta, \alpha) A_{1}, \\
D_{2} & =K(\kappa, \eta, \alpha) A_{1},
\end{aligned}
$$

where $A_{1}$ can be figured out from the initial condition $(t=0)$. Other functions, which depends on $\kappa, \eta$, and $\alpha$ are written in the Appendix. To visualize the mode shapes, $A_{1}=1$ is usually used.

\section{RESULTS}

The mode shapes of T-shaped $(\kappa<1)$ and overhang-shaped $(\kappa>1)$ cantilevers are shown in Fig. 2. For T-shaped cantilevers $(\kappa=0.5)$, the first (a) and second (c) modes show a smoother deflection, see red dashed line of $\eta=0.1$, blue dotted line of $\eta=0.3$, and green dash-dotted line of $\eta=0.5$ in comparison to the deflection of a uniform (rectangular) cantilever (black solid line). It's worth to noting that we are fixing the deflection at the transition position, $x=L_{t} / L=\eta L$, of the Tand overhanged-shaped cantilever to be the same as that of the uniform cantilever. Therefore, if we normalize all mode shapes using a maximal deflection at $x=L$ as a referenced scale, the T-shaped cantilever will present flatter lines. These lines will be too close and it is difficult to distinguish them from each other. Hence, we choose the deflection at the transition points (for example, $\eta=$ 0.1 presented by red dashed line and red dashed arrows) as the normalized scale. As a result, the 

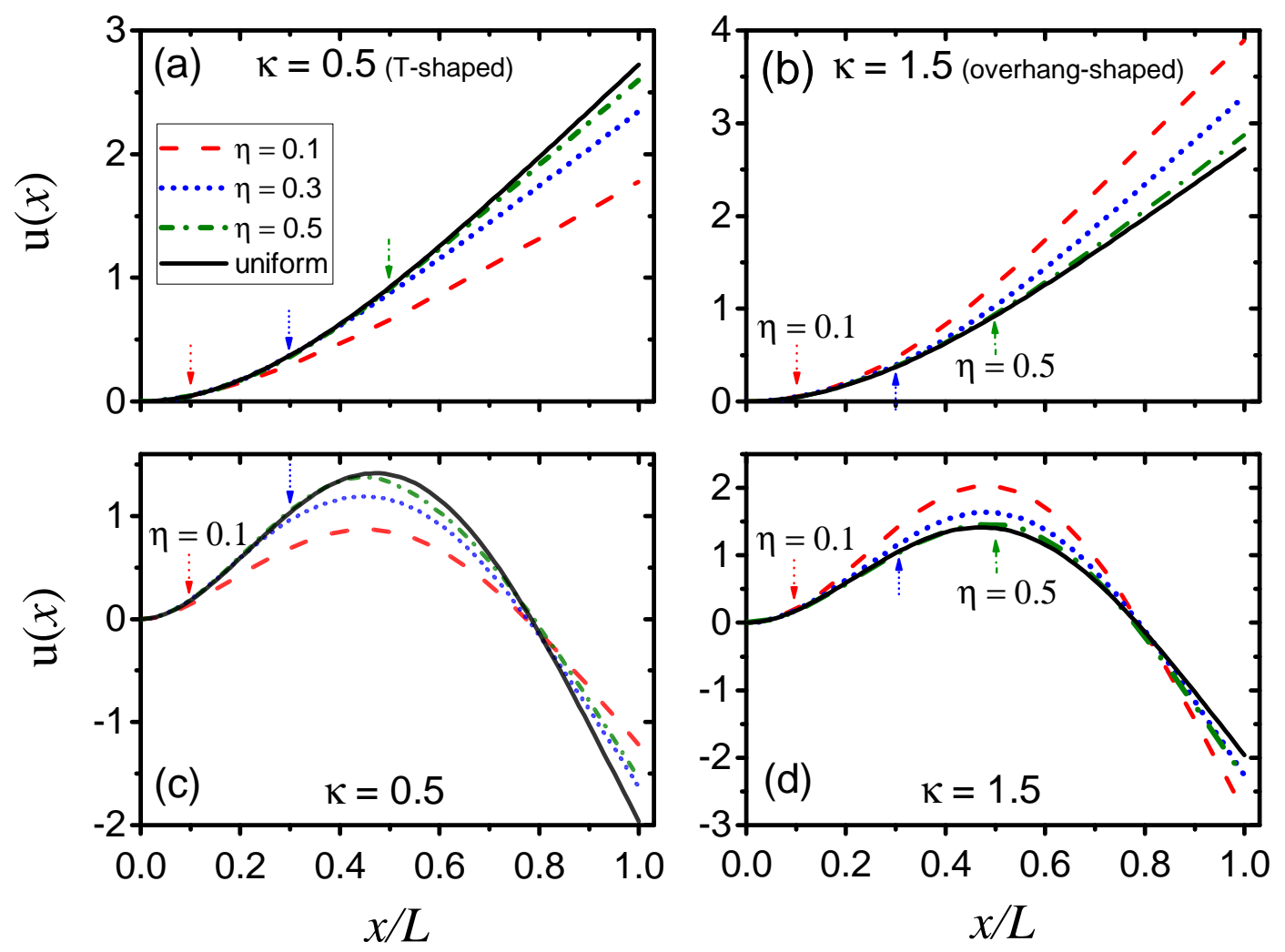

Fig. 2. Correct mode shapes of the first [(a) and (b)] and second [(c) and (d)] modes of T- and overhang-shaped cantilevers in comparison to the mode shapes (black solid lines) of a uniform cantilever. The arrows pointed out the position the two modeshapes start deviating each other corresponding to the overhang length, $\eta=0.1$ (red dashed), 0.3 (blue dotted), and 0.5 (green dash-dotted lines).

maximal points of the mode shapes are different. This is because the smaller width of the clamped position has soften the T-shaped cantilever, i.e. reduce the inertia moment $I$ in Eq. (1).

Having the correct solutions of Eq. (13), we obtained the corresponding frequencies $(f)$ of several modes. Using $\eta=0.1\left(=L_{t} / L\right)$ as an example, the results from the solution of Eq. (13) is confirmed by that from FEM (finite element method, COMSOL Multiphysics) simulations and the two results are shown in Fig. 3 where frequencies of the first two modes are presented. Comparing the frequencies from the two calculation methods, we saw that the greatest deviation is not greater than 3\% (similar behavior of the third and fouth modes are seen and not shown here). In general, change in frequency was seen when $\kappa \neq 1$, i.e., $f$ increases with $\kappa$. This is because the T-shaped cantilever is "softer" and the ovehang-shaped cantilever is "harder" than the uniform cantilever. Analytically, T-shaped cantilever with smaller width has been elongated to an "effective" length; therefore, reduced the frequency (see Eq. (2)).

As a result, the approximate mode shapes commonly used in previous studies from a uniform cantilever, could be used in a very limited case. When the width of the undercut (or overhang) 
Table 2. The solution $\alpha$ from Eq. (13).

\begin{tabular}{cccccc}
\hline \hline & & \multicolumn{4}{c}{ First four roots } \\
\cline { 3 - 6 } & $\eta=L_{t} / L_{c}$ & mode 1 & mode 2 & mode 3 & mode 4 \\
\hline \multirow{2}{*}{ T-shaped cantilever, } & 0.1 & 1.739 & 4.503 & 7.656 & 10.814 \\
$\kappa=0.5$ & 0.2 & 1.666 & 4.506 & 7.698 & 10.877 \\
& 0.3 & 1.625 & 4.526 & 7.752 & 11.016 \\
& 0.4 & 1.604 & 4.532 & 7.861 & 11.038 \\
\hline Uniform beam, $\kappa=1$ & 0 or 1 & 1.875 & 4.694 & 7.854 & 10.995 \\
\hline \multirow{2}{*}{ Overhang-shaped cantilever, } & 0.1 & 1.933 & 4.804 & 7.992 & 11.126 \\
$\kappa=1.5$ & 0.2 & 1.982 & 4.829 & 7.939 & 11.012 \\
& 0.3 & 2.019 & 4.794 & 7.86 & 10.999 \\
\hline \hline
\end{tabular}
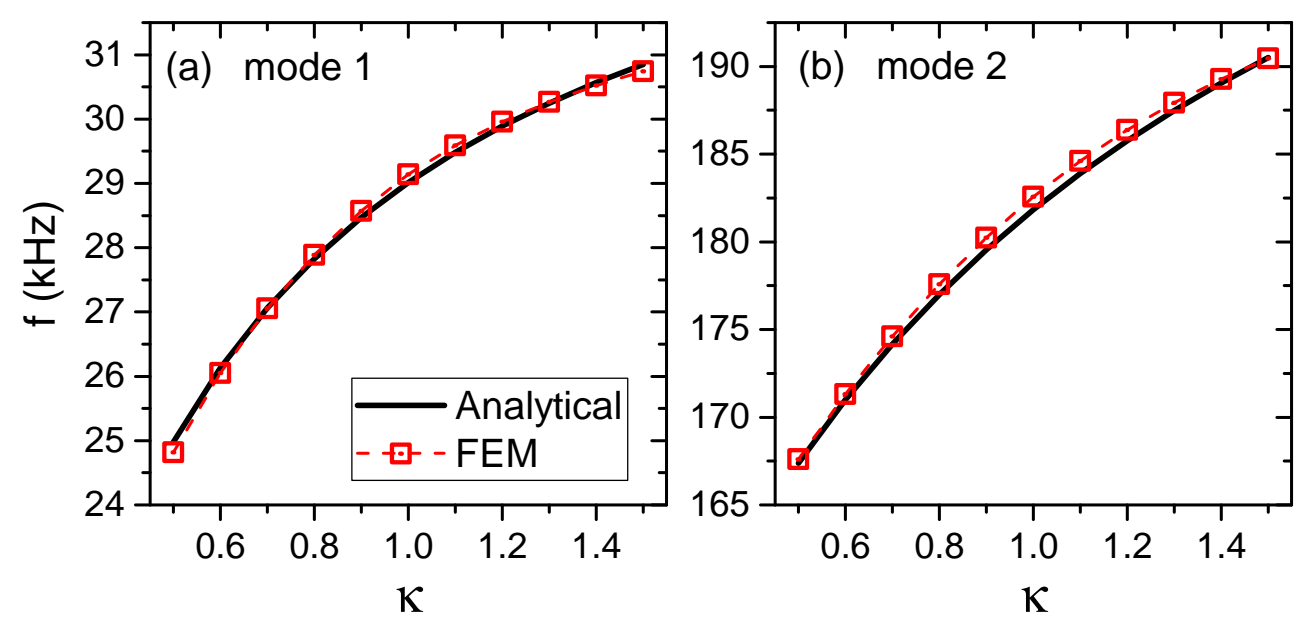

Fig. 3. Frequency of the first and second mode obtained from Eq. (13) (black solid line) and from FEM simulations (red squares). The deviation between the two method is not greater than $3 \%$. Here, the parameters in Table 3 are used.

is much greater than that of the cantilever, the frequency could be significantly varied, as shown in Fig. 4. In Table 2, the frequencies of the first four modes are shown which presented the increase of $f$ with $\kappa$ and these $f$ s are significantly different from the value of the uniform cantilever (shown by black solid lines). Changing the length of the overhanging part, $\eta=0-1$, the first mode (a) has one minimum if $\kappa<1$ (black rounds) and one maximum if $\kappa>1$ (red squares). The corresponding number of minima and maxima of the second mode is two, as could be seen in Fig. 4(b). 
Table 3. Parameters for calculations.

\begin{tabular}{lll}
\hline Parameters & Symbol (Unit) & $\mathrm{Si}_{3} \mathrm{~N}_{4}$ \\
\hline \hline Length & $L(\mu \mathrm{m})$ & 200 \\
Thickness & $t(\mu \mathrm{m})$ & 0.8 \\
Young's modulus & $E(\mathrm{GPa})$ & 250 \\
Density & $\rho\left(\mathrm{kg} / \mathrm{m}^{3}\right)$ & 3100 \\
\hline
\end{tabular}

Therefore, the frequency is strongly dependent on the width and length of the overhanging part. This suggests that, using of correct mode shapes is significantly important for obtaining the correct frequencies of the width-varying cantilevers.
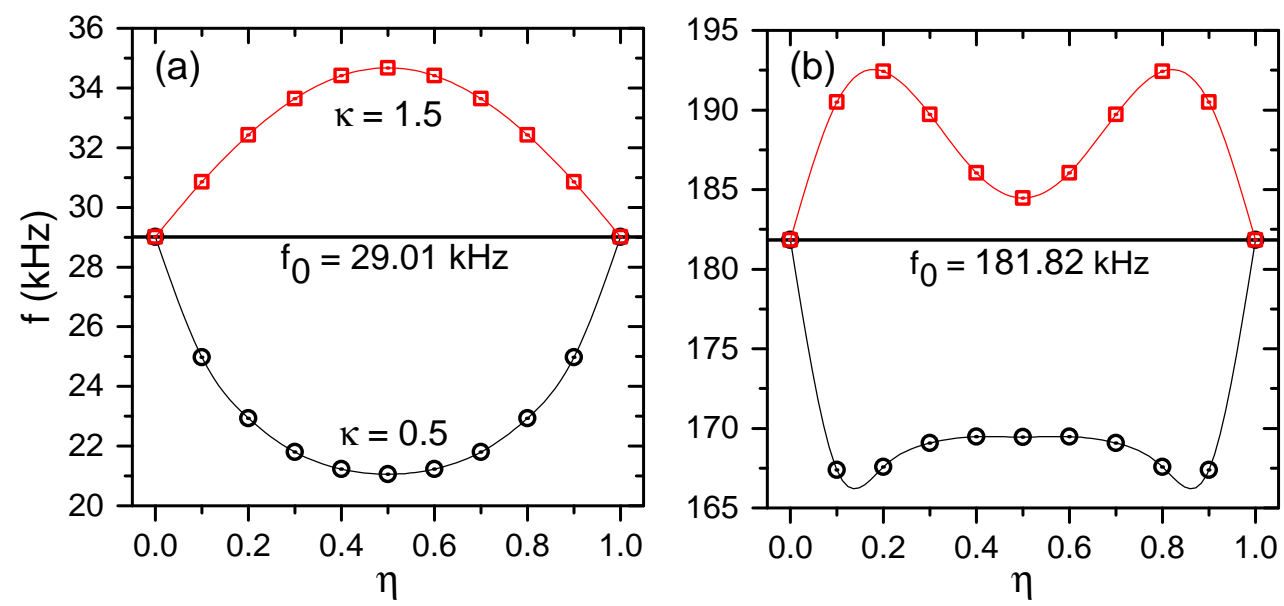

Fig. 4. Frequency of the first (a) and second (b) mode in comparison to that from a rectangular cantilever (black solid line). T-shaped[overhang-shaped] cantilevers have frequency minima[maxima] and the number of minima[maxima] is corresponding to the mode number.

\section{CONCLUSIONS}

In this study, we performed a detailed calculation for the exact mode shapes and frequencies of varying width cantilevers, the T- and overhang-shaped cantilevers. It was seen that the mode shapes of a uniform rectangular cantilever should be carefully used when the cantilever is extended to that of varying widths. If the width of the overhanging part is greater than 1.1 times the width of the main cantilever, the correct mode shapes should be used. The obtained analytical results could be used for cantilever with similar geometric widths. The analytical results have been confirmed by the finite element method simulations with a very low deviation. This study gave important contributions in the analysis of mode shapes and frequencies of micro-mechanical systems of involved dimensions. 


\section{ACKNOWLEDGMENT}

We are thankful to Prof. Van-Hoang Le (HCMUE) for encouragement. This research is funded by Vietnam National Foundation for Science and Technology Development (NAFOSTED) under grant number 103.01-2019.345.

\section{REFERENCES}

[1] C. H. Metzger and K. Karrai, Nature 432 (2004) 1002.

[2] T. Corbitt, D. Ottaway, E. Innerhofer, J. Pelc and N. Mavalvala, Phys. Rev. A 74 (2006) 021802(R).

[3] P. Rabl, S. J. Kolkowitz, F. H. L. Koppens, J. G. E. Harris, P. Zoller and M. D. Lukin, Nat. Phys. 6 (2010) 602.

[4] N. D. Vy, L. T. Dat and T. Iida, Appl. Phys. Lett. 109 (2016) 054102.

[5] J. Fritz, M. K. Baller, H. P. Lang, H. Rothuizen, P. Vettiger, E. Meyer, H. J. Güntherodt, C. Gerber and J. K. Gimzewski, Science 288 (2000) 316.

[6] B. Ilic, D. Czaplewski, H. G. Craighead, P. Neuzil, C. Campagnolo and C. Batt, Appl. Phys. Lett. 77 (2000) 450.

[7] B. Ilic, D. Czaplewski, M. Zalalutdinov, H. G. Craighead, P. Neuzil, C. Campagnolo and C. Batt, J. Vac. Sci. Technol. B - Microelectron. Nanometer. Struct. Process. Meas. Phenom. 19 (2001) 2825.

[8] F. Huber, H. P. Lang, J. Zhang, D. Rimoldi and C. Gerber, Swiss medical weekly 145 (2015) .

[9] C. A. Savran, S. M. Knudsen, A. D. Ellington and S. R. Manalis, Anal. Chem. 76 (2004) 3194.

[10] S.-J. Hyun, H.-S. Kim, Y.-J. Kim and H.-I. Jung, Sens. Actuators B: Chem. 117 (2006) 415.

[11] P. Datskos, S. Rajic and I. Datskou, Ultramicroscopy 82 (2000) 49.

[12] L. T. Dat, H. T. Huy and N. D. Vy, Commun. in Physics 28 (2018) 255.

[13] T. Thundat, R. J. Warmack, G. Y. Chen and D. P. Allison, Appl. Phys. Lett. 64 (1994) 2894.

[14] S. Guillon, D. Saya, L. Mazenq, S. Perisanu, P. Vincent, A. Lazarus, O. Thomas and L. Nicu, Nanotechnology 22 (2011) 245501.

[15] J. A. Plaza, K. Zinoviev, G. Villanueva, M. Álvarez, J. Tamayo, C. Domínguez and L. M. Lechuga, Appl. Phys. Lett. 89 (2006) 094109.

[16] S.-D. Kwon, Appl. Phys. Lett. 97 (2010) 164102.

[17] J. E. Sader, Rev. Sci. Instrum. 66 (1995) 4583.

[18] J. E. Sader, J. A. Sanelli, B. D. Adamson, J. P. Monty, X. Wei, S. A. Crawford, J. R. Friend, I. Marusic, P. Mulvaney and E. J. Bieske, Rev. Sci. Instrum. 83 (2012) 103705.

[19] G. Zhang, L. Zhao, Z. Jiang, S. Yang, Y. Zhao, E. Huang, X. Wang and Z. Liu, J. Phys. D: Appl. Phys. 44 (2011) 425402.

[20] N. D. Vy, N. V. Cuong and C. M. Hoang, J. Mecha. 35 (2018) 351.

[21] S. Timoshenko and D. Young, Engineering mechanics: Statics, no. v. 1, McGraw-Hill Book Company, Inc., 1937.

\section{APPENDIX}

The explicit form of factors in Eqs. (15)-(16) are shown as follows,

$$
\Gamma(\kappa, \eta, \alpha)=\frac{\Phi(\kappa, \eta, \alpha)}{\Psi(\kappa, \eta, \alpha)}
$$

where,

$$
\begin{aligned}
\Phi(\kappa, \eta, \alpha)= & -2(\kappa+1) \operatorname{csch} \eta \alpha(\sin \alpha+\sinh \alpha)-2(\kappa-1)\{\cos (\alpha-\eta \alpha)-\sinh \alpha \sin (\eta \alpha) \\
& +\operatorname{coth}(\eta \alpha)[\sin (\alpha-\eta \alpha)+\sinh \alpha \cos (\eta \alpha)]\}+ \\
& +(\kappa-1) \cosh \alpha \sec (\eta \alpha)[\cos (2 \eta \alpha)-\sin (2 \eta \alpha) \operatorname{coth}(\eta \alpha)+1],
\end{aligned}
$$




$$
\begin{aligned}
\Psi(\kappa, \eta, \alpha)= & 2\{(\kappa+1) \operatorname{csch}(\eta \alpha)(\cos \alpha+\cosh \alpha)+ \\
& +(\kappa-1)[\sin (\alpha-\eta \alpha)-\sinh \alpha \cos \eta \alpha+\cosh \alpha \sin \eta \alpha]+ \\
& +(\kappa-1) \operatorname{coth} \eta \alpha+[\sin \eta \alpha(\sin \alpha-\sinh \alpha)+\cos \eta \alpha(\cos \alpha+\cosh \alpha)]\} \\
H(\kappa, \eta, \alpha)= & \left.\frac{1}{2}(-(1-\kappa) \sinh \eta \alpha(\Gamma \cos \eta \alpha+\sin \eta \alpha)+\cosh \eta \alpha(\Gamma \sin \eta \alpha+\cos \eta \alpha))+\kappa+1\right) \\
E(\kappa, \eta, \alpha)= & \frac{1}{2}((\kappa+1)(\Gamma(\kappa, \eta, \alpha)+\tan \eta \alpha)-(1-\kappa)(\sec (\eta \alpha) \cosh \eta \alpha \Gamma(\kappa, \eta, \alpha)+ \\
& +\sec (\eta \alpha) \sinh (\eta \alpha))-2 \tan (\eta \alpha) H(\kappa, \eta, \alpha)) \\
\Omega(\kappa, \eta, \alpha)= & \frac{1}{2}(-(\kappa+1)(\operatorname{coth}(\eta \alpha) \Gamma(\kappa, \eta, \alpha)+1)-(\kappa-1) \operatorname{csch}(\eta \alpha)(\cos (\eta \alpha) \Gamma(\kappa, \eta, \alpha)+ \\
& +\sin (\eta \alpha))-2 \operatorname{coth}(\eta \alpha) K(\kappa, \eta, \alpha)) \\
& 1 \\
& +\sin (\eta \alpha))-(\kappa+1) \Gamma(\kappa, \eta, \alpha)) \\
K(\kappa, \eta, \alpha)= & ((\kappa-1)(\sinh (\eta \alpha)(\cos (\eta \alpha)-\sin (\eta \alpha) \Gamma(\kappa, \eta, \alpha))-\cosh (\eta \alpha)(\cos (\eta \alpha) \Gamma(\kappa, \eta, \alpha)+
\end{aligned}
$$

